\title{
Efecto del estiramiento mantenido versus el estiramiento mantenido previa aplicación de ultrasonido sobre la extensibilidad de los músculos isquiotibiales en mujeres adultas
}

\author{
Effect of stretching versus stretching after application of ultrasound on the exten- \\ sibility of the hamstring muscles in adult women
}

Carolina Anaya Niño', Adriana Angarita Fonseca², German Andrés Villamizar Rayón ${ }^{3}$

Para citar este artículo: Anaya Niño C, Angarita Fonseca A, Villamizar Rayón GA. Efecto del estiramiento mantenido versus el estiramiento mantenido previa aplicación de ultrasonido sobre la extensibilidad de los músculos isquiotibiales en mujeres adultas. UstaSalud. 2017;16: 35-42

Licencia Creative Commons

\section{(c) (1) () $\Theta$} lo tanto, los lectores pueden acceder libremente a los artículos en su formato .pdf, igualmente podrán descargarlos y difundirlos; sin embargo no podrán modificarlos o alterarlos, adicionalmente se debe reconocer la autoría de las personas que figuran en las publicaciones, pero estas no podrán comercializadas.

\section{RESUMEN}

Objetivo: Comparar los efectos del estiramiento mantenido, versus el estiramiento mantenido, previo calentamiento con ultrasonido terapéutico sobre la extensibilidad de los músculos isquiotibiales en mujeres universitarias de 18 a 30 años de edad.

Metodología: Se llevo a cabo un ensayo clínico controlado aleatorizado. Catorce mujeres $(22,3 \pm 3,9$ años) fueron asignadas aleatoriamente a dos grupos, EM ( 7 minutos) previo US (US+EM), $n=8$ y EM por 7 minutos, $\mathrm{n}=6$. Las diferencias entre evaluaciones se compararon mediante la prueba t de student; el efecto de la intervención se evalúo mediante un análisis de covarianza.

Resultados: Aumentó significativamente la flexibilidad de miembros inferiores en los dos grupos. En el grupo US+EM aumentó $-8,75 \pm 5,5$; mientras que en el grupo EM el aumento fue -3,5 $\pm 2,7$; al comparar los grupos no se encontraron diferencias estadísticamente significativas. Se encontró un aumento estadísticamente significativo en los grados finales en el grupo intervenido US+EM, -5,8(IC95\%:-10,9;-0,67p=0,030) en comparación con el grupo de EM ajustando solo por puntaje inicial.

Conclusión: Una sola exposición al US+EM es suficiente para potenciar las ganancias en la extensibilidad de los músculos isquiotibiales en mujeres adultas comparado con el EM.

Palabras clave: Terapia por ultrasonido, estiramiento, flexibilidad, extensibilidad, fisioterapia.

\section{ABSTRACT}

Objective: To compare the effects of SM versus US + SM on the extensibility of the hamstring muscles in adult women.

Methods: A Randomized Controlled Clinical Trial was conducted. Fourteen women (22.3 \pm 3.9 years) randomly assigned to two groups, SM ( 7 minutes) prior US (US $+S M), n=8$ and SM for 7 minutes, $n=6$. The differences between assessments were compared using student's t-test; the effect of the intervention was evaluated by analysis of covariance.

Results: Significantly increased flexibility of lower limbs in both groups. In the US + SM group increased -8.75 \pm 5.5 ; while increasing in the SM group was $-3.5 \pm 2.7$; when comparing the groups, no statistically significant differences were found. A statistically significant increase was found in final grades in the US + SM intervention group, $-5.8(95 \% \mathrm{Cl}:-10.9 ;-0.67 \mathrm{p}=0.030)$ compared with the SM group adjusting only for baseline score.

Conclusion: A single exposure to US + SM is sufficient to enhance the gains in hamstring extensibility in adult women compared to SM.

Keywords: Ultrasound, stretching, flexibility, extensibility, physiotherapy.
'Fisioterapeuta, especialista en Docencia Universitaria y magíster (c) en Fisioterapia por la Universidad Industrial de Santander.

${ }^{2}$ Fisioterapeuta, magíster en Epidemiología Grupo de investigación CliniUDES, Universidad de Santander.

${ }^{3}$ Médico, Universidad de Santander.

Autor de correspondencia:

Carolina Anaya Niño

Correo electrónico:

caritoanaya@gmail.com
Recibido para publicación: 26 de noviembre de 2016. Aceptado para publicación: 16 de enero de 2017. 


\section{INTRODUCCIÓN}

En la literatura es común encontrar el uso de los términos extensibilidad y flexibilidad de forma no diferenciada; sin embargo, los términos hacen referencia a características diferentes. En primer lugar, la extensibilidad de los tejidos es definida como la capacidad de un músculo o unión tendinosa de estirarse a su máxima longitud ${ }^{1,2}$; en tanto que la flexibilidad se define como la amplitud de movimiento disponible en una articulación o grupo de articulaciones ${ }^{2-4}$. Por otro lado, las retracciones musculares resultan en una disminución de la extensibilidad de los tejidos y, por ende, de la movilidad articular ${ }^{2}$. Por esta razón, diversos métodos de fisioterapia estan dirigidos para prevenir la deformidad y la discapacidad resultante de las retracciones musculares. Dentro de dichos métodos se incluyen: la aplicación de crioterapia, modalidades de calentamiento superficial o profundo, estiramiento mantenido, dispositivos de movimiento pasivo continuo, ejercicios activos o pasivos; los cuales pueden emplearse de forma aislada o combinados.

Wang et al. ${ }^{5}$ en 1991 establecieron que la regulación de la extensibilidad de los tejidos puede estar influenciada por la madurez de las células musculoesqueleticas, las cuales controlan y modulan la rigidez y el límite elástico de los tejidos, a través de la expresión selectiva de las isoformas de titina; de esta forma los programas de entrenamiento de la flexibilidad deberían tener en cuenta estas características para ser efectivos; sin embargo, no existe claridad de la forma correcta de realizarlo.

Otras características importantes que exhiben las fibras musculares son la elasticidad y la plasticidad. La primera se refiere a la tendencia de un tejido de retornar a su longitud de reposo después de la aplicación de un estiramiento pasivo ${ }^{1,2,6}$; mientras que la plasticidad es la tendencia de un tejido a asumir una nueva y mayor longitud después de un estiramiento pasivo, propiedad que garantiza al tejido musculotendinoso al asumir una longitud permanente después de programas de flexibilización ${ }^{2,7,8}$.

La significancia clínica de la plasticidad en el tratamiento de fisioterapia se dirige hacia los cambios en la longitud muscular que pueden ser logrados mediante programas de flexibilización, adecuadamente instau- rados; en los cuales el terapeuta tiene la autonomía de seleccionar las modalidades de intervención que tengan mayor evidencia para su logro.

En estudios animales se ha establecido que la combinación del estiramiento mantenido con el calor profundo, es un método efectivo para mejorar la extensibilidad de músculos y tendones; dentro de estos estudios cabe resaltar los realizados por Usuba et al. ${ }^{9}$ en 2006, quienes encontraron que después de seis sesiones de tratamiento durante dos semanas realizadas a 93 ratas Wistar con contractura en flexión de rodilla, la aplicación de estiramiento mantenido combinado con cualquiera de las dos modalidades de calentamiento (infrarrojo o ultrasonido), es más efectiva para mejorar el rango de movimiento y para disminuir la fase de latencia de las contracturas moderadas a severas.

Sin embargo, en seres humanos existe poca evidencia que establezca la relación entre el calor profundo y el estiramiento mantenido; dentro de los reportes que han encontrado asociación se encuentra el realizado por Wessling et al. ${ }^{2}$ en la extensibilidad del tríceps sural. De otro lado, Brodowics et al. ${ }^{10}$ en 1996 reportan resultados inconclusos en relación con la aplicación previa al estiramiento de crioterapia o paquete caliente.

Ahora bien, la hipótesis que el estiramiento mantenido, previa aplicación de ultrasonido terapéutico, es más efectivo que el solo estiramiento sobre la extensibilidad de los músculos isquiotibiales en mujeres universitarias, puede estar fundamentada en que el ultrasonido es una modalidad de calor profundo que bajo parámetros establecidos de intensidad, duración y frecuencia puede tener efectos mecánicos o térmicos. Gersten citado por Wessling et al. ${ }^{2}$ demostró que la extensibilidad del tendón aumenta significativamente con la aplicación del ultrasonido entre 1 a $3 \mathrm{~W} /$ $\mathrm{cm}^{2}$, así mismo, la intensidad del US se encuentra en relación con la profundidad del tejido.

Por lo anterior, el objetivo de este estudio fue comparar los efectos del estiramiento mantenido, versus el estiramiento mantenido, previo calentamiento con ultrasonido terapéutico sobre la extensibilidad de los músculos isquiotibiales en mujeres universitarias de 18 a 30 años de edad. 


\section{MATERIALES Y MÉTODOS}

Se ejecutó un ensayo clínico aleatorizado controlado en una muestra de 14 mujeres seleccionadas a conveniencia, las participantes eran estudiantes de fisioterapia de una universidad en Bucaramanga, Santander, quienes decidieron participar voluntariamente en el estudio.

Según la Resolución 008430 de 1993 del Ministerio de Salud de Colombia esta investigación se clasifica en la categoría de Investigación con Riesgo Mínimo, debido a que se emplean procedimientos comunes en el actuar del fisioterapeuta, empleando parámetros seguros. Adicionalmente, se garantizó el respeto a los principios éticos fundamentales de autonomía, no maleficencia, beneficencia y justicia durante la ejecución del estudio y durante el manejo de la información se garantiza la confidencialidad. El estudio fue previamente aprobado por el Comité de Investigación del Programa de Fisioterapia de la Universidad de Santander; además se obtuvo el consentimiento informado por escrito de cada participante.

Para participar en esta investigación los participantes debian tener entre 18 y 30 años, no haber sufrido trauma o fractura en sus extremidades inferiores, tener el índice de masa corporal (IMC) entre 18 y 24,9, no estar en estado de embarazo y tampoco participar en programas de acondicionamiento físico durante el desarrollo de la investigación.

El evaluador A se encargó de realizar la asignación aleatoria de las participantes a los grupos de intervención mediante sobres sellados.

\section{Intervención}

US+EM: El investigador A aplicó ultrasonido con una frecuencia de $1 \mathrm{MHz}$, intensidad $1,5 \mathrm{~W} / \mathrm{cm}^{2} \mathrm{mo}-$ dalidad continua durante 8 minutos sobre la zona del vientre muscular de los isquiotibiales ${ }^{11}$ (Figura 1). Posteriormente, el investigador $\mathrm{B}$ realizó el estiramiento mantenido de los músculos isquiotibiales durante 7 minutos, el sujeto fue ubicado en la misma posición en la cual se realizó la evaluación de la flexibilidad.

EM: Después de permanecer en posición prona durante 8 minutos, el investigador $\mathrm{B}$ realizó el estira- miento mantenido de los músculos isquiotibiales durante 7 minutos, en las mismas condiciones del grupo $\mathrm{EM}+\mathrm{US}$.

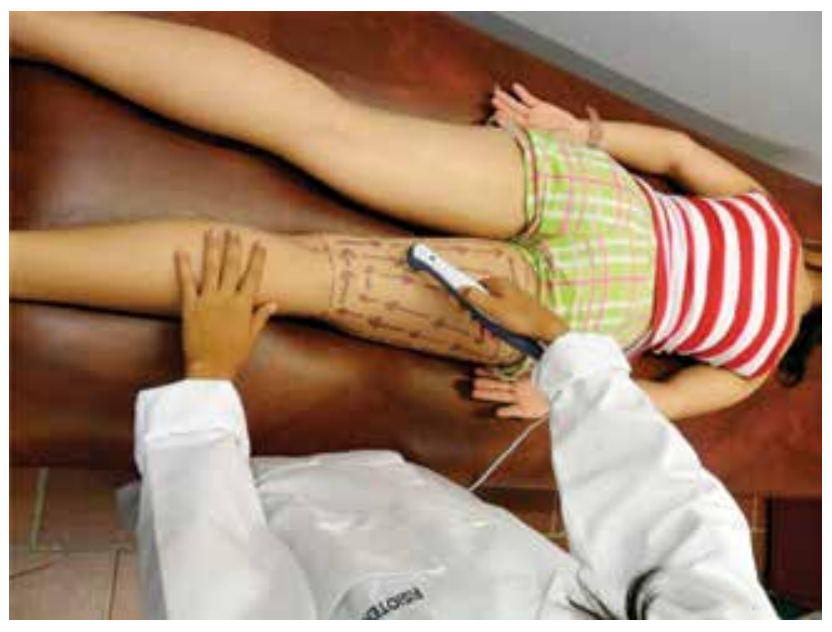

Figura 1. Zona de aplicación del ultrasonido.

El estiramiento en ambos grupos fue realizado por el mismo investigador con el objetivo de mantener la estandarización en cuanto a duración, intensidad, frecuencia y técnica de estiramiento.

Arco de movilidad articular de extensión de rodilla con 90 grados de flexión de cadera como lo propuesto por Norkin y White ${ }^{12}$ (Figura 2). El Evaluador B realizó las mediciones en el miembro inferior derecho de todas las participantes.

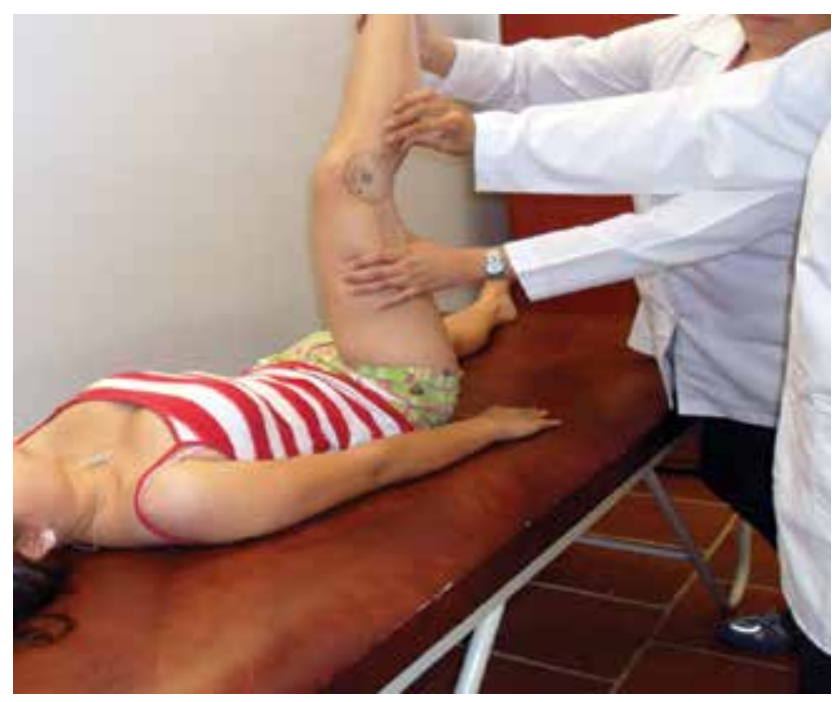

Figura 2. Medición de la flexibilidad de los músculos isquiotibiales. 
Las mediciones se hicieron antes e inmediatamente después de la intervención por el mismo evaluador, mediante un goniómetro universal marca Prestige $\mathrm{Medical}^{\circ}$, para esto se ubicó a las participantes en posición supino y se utilizaron marcas en cóndilo lateral del fémur, trocánter mayor y maléolo lateral de la tibia para garantizar los mismos puntos de referencia entre las pruebas; para la medición la cadera y la rodilla se mantuvieron en 90 grados de flexión, el eje se ubicó en el cóndilo lateral del fémur, el brazo fijo se proyectó con la diáfisis del fémur hacia el trocánter mayor y el brazo móvil hacia el maléolo lateral de la tibia. Las mediciones fueron realizadas pasivamente moviendo la extremidad hacia la posición terminal de extensión de rodilla; definida como el punto en el que el sujeto refiere una sensación de incomodidad a nivel de los isquiotibiales y percibido por el evaluador como una resistencia al estiramiento. Este evaluador fue cegado al grupo asignado para cada participante para evitar sesgos en los resultados.

Los cuatro evaluadores hicieron un entrenamiento previo de los procedimientos que se deberían seguir, con el objetivo de estandarizarlos.

Se obtuvo la información sociodemográfica de las participantes, así como de los datos de talla y peso para la obtención del IMC.

El nivel de actividad física (AF) fue establecido mediante el IPAQ, el cual es un instrumento diseñado principalmente para la vigilancia del nivel de AF en la población adulta en un período de recuerdo de siete días. La versión larga del IPAQ evalúa la AF realizada en diferentes contextos que incluyen el tiempo libre, actividades domésticas y actividades en el jardín, actividades de desplazamiento y actividades relacionadas con el trabajo. La versión corta del IPAQ pregunta sobre tres tipos específicos de actividad realizada en los tres dominios, incluyendo el tiempo que gasta en las actividades en sedente. Se indaga por la AF de caminata, AF moderada y AF vigorosa; la frecuencia (medida en días por semana) y duración (tiempo por día). De esta manera el IPAQ corto proporciona la evaluación separada de la AF de caminata y AFM y AFV, así como también un resultado total que describe el nivel global de la AF. El cómputo total requiere la suma de la duración (en minutos) y la frecuencia (en días) de la caminata, AFM y AFV ${ }^{13}$.
Para el IPAQ, una persona se clasifica en la categoría AF alta si cumple con alguno de los siguientes criterios: 1) Al menos tres días a la semana de AFV alcanzando un mínimo de $1.500 \mathrm{MET}-\mathrm{min} / \mathrm{sem}$. 2) Siete días de cualquier combinación de caminata, AFM o AFV hasta lograr un mínimo de al menos 3000 MET$\mathrm{min} / \mathrm{sem}$. Se considera en la categoría AF moderada si cumple con: 1) Tres o más días de AFV al menos 20 min por día; o 2) Cinco o más días de AFM o caminar al menos 30 min por día; o 3) Cinco o más días de cualquier combinación de caminata, AFM o AFV hasta lograr un mínimo de al menos 600 MET-min/ sem. Se clasifica en la categoría AF Baja la persona que no cumpla con los criterios para estar en alguna de las dos anteriores ${ }^{13}$.

\section{Análisis estadístico}

Se describió la población de estudio de acuerdo con sus características sociodemográficas, aplicando medidas de tendencia central y dispersión, según la naturaleza y distribución de cada variable; así, después de comprobar la normalidad de las variables con la prueba de Shapiro Wilk se realizó el análisis univariado a través de promedios, medias y desviación estándar. Posteriormente, se hizo un análisis bivariado mediante la diferencia de medias con la prueba $t$ de student y finalmente, se aplicó un análisis multivariado mediante un análisis de covarianza ANCOVA, donde la variable dependiente fue el puntaje final, ajustado por el puntaje inicial y el grupo de intervención y la actividad física previa. Para todo el análisis se consideró un nivel de significancia 0,05, la base de datos se realizó en el programa Microsoft Office Excel $^{\circledast}$ (Excel 2013; Microsoft Corporation, Redmont: WA, USA) y el análisis con el paquete estadístico STATA $11^{\otimes}$ (StataCorp. 2009. Stata Statistical Software: Release 11. College Station, TX: StataCorp LP).

\section{RESULTADOS}

La muestra (Figura 3) estuvo conformada por 14 mujeres con promedio de edad de 22,3 $\pm 3,9$ años $\mathrm{y}$ un IMC de $20,7 \pm 2,1 \mathrm{~kg} / \mathrm{m} 2$; distribuidas en dos grupos: 8 mujeres $(23,3 \pm 3,4$ años $)$ quienes recibieron estiramiento mantenido y ultrasonido (EM+US) y las 6 restantes ( $21 \pm 4,5$ años) asignadas al grupo de estiramiento mantenido (EM). No se encontraron diferencias estadísticamente significativas en edad, IMC y nivel de actividad física (Tabla 1). 


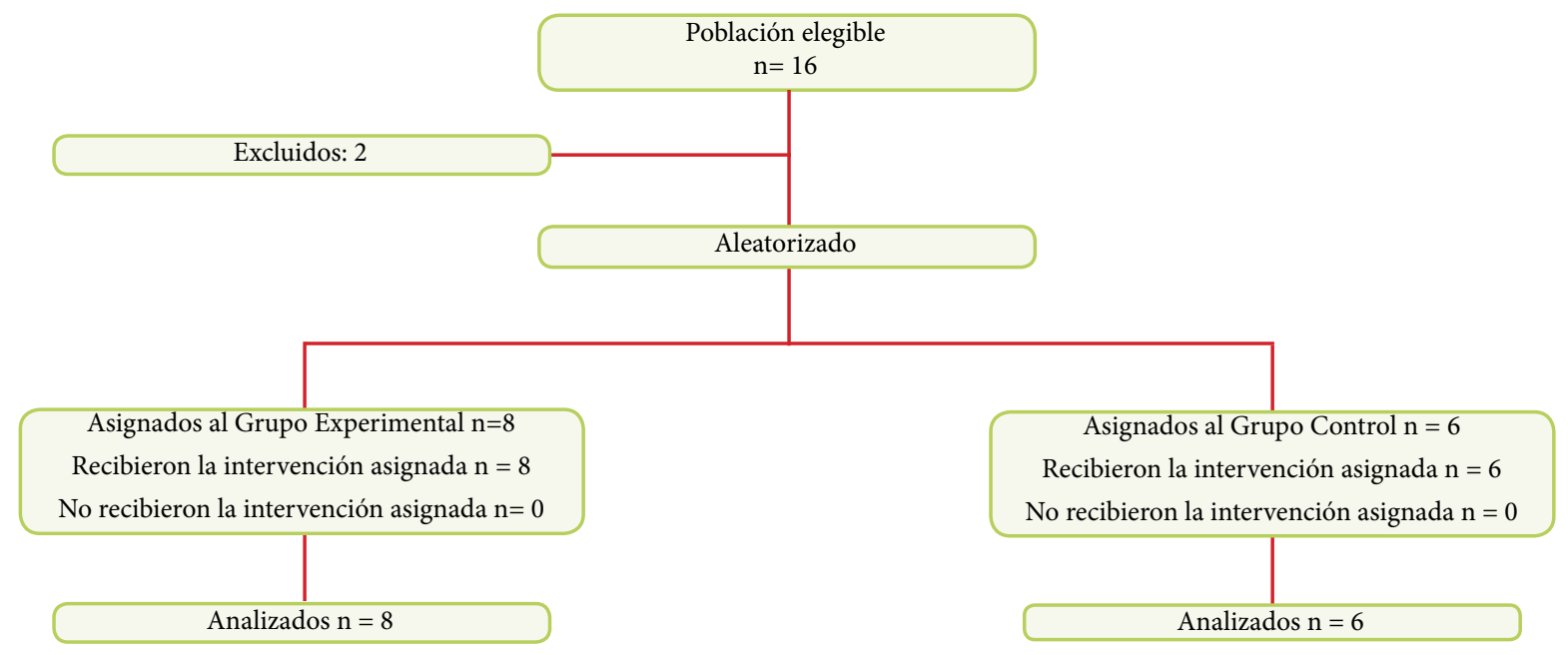

Figura 3. Flujograma de estudio.

Tabla 1. Características sociodemográficas de la población

\begin{tabular}{llll}
\hline Variable & $\begin{array}{c}\text { Estiramiento } \\
\text { Mantenido y } \\
\text { Ultrasonido }\end{array}$ & $\begin{array}{c}\text { Estiramiento } \\
\text { Mantenido }\end{array}$ & Valor de p \\
\hline Edad & $23,4 \pm 3,4$ & $21,0 \pm 4,5$ & 0,281 \\
IMC & $20,8 \pm 2,1$ & $20,5 \pm 2,2$ & 0,790 \\
\hline Nivel de actividad física & & \\
Sedentario & $4(50,0)$ & $4(66,7)$ & 0,120 \\
Baja & $0(0,0)$ & $2(33,3)$ & \\
Moderada & $2(25,0)$ & $0(0,0)$ & \\
Alta & $2(25,0)$ & $0(0,0)$ & \\
\hline
\end{tabular}

Se encontró que en ambos grupos aumentó significativamente la flexibilidad de miembros inferiores, en el grupo de EM + US hubo un aumento de -8,75 \pm 5,5; mientras que en el Grupo de EM el aumento fue de $-3,5 \pm 2,7$ (Tabla 2); al comparar el aumento de un grupo con el otro no se encontraron diferencias estadísticamente significativas (Figura 4).

Tabla 2. Cambios en la medición goniométrica entre los grupos antes y después de la intervención

\begin{tabular}{ccccc}
\hline \multicolumn{1}{c}{ Grupo } & Pretest & Postest & Diferencia & Valor $\mathbf{p}$ \\
\hline $\begin{array}{l}\text { Estiramiento man- } \\
\text { tenido y ultraso- } \\
\text { nido* }\end{array}$ & $37 \pm 11,6$ & $28,3 \pm 10,3$ & $-8,75 \pm 5,5$ & 0,003 \\
$\begin{array}{l}\text { Estiramiento man- } \\
\text { tenido* }\end{array}$ & $39,8 \pm 7,1$ & $36,3 \pm 6,7$ & $-3,5 \pm 2,7$ & 0,024 \\
\hline
\end{tabular}

*Valor p comparando promedio de las diferencias del grupo estiramiento más ultrasonido vs ultrasonido fue de 0,0525 .

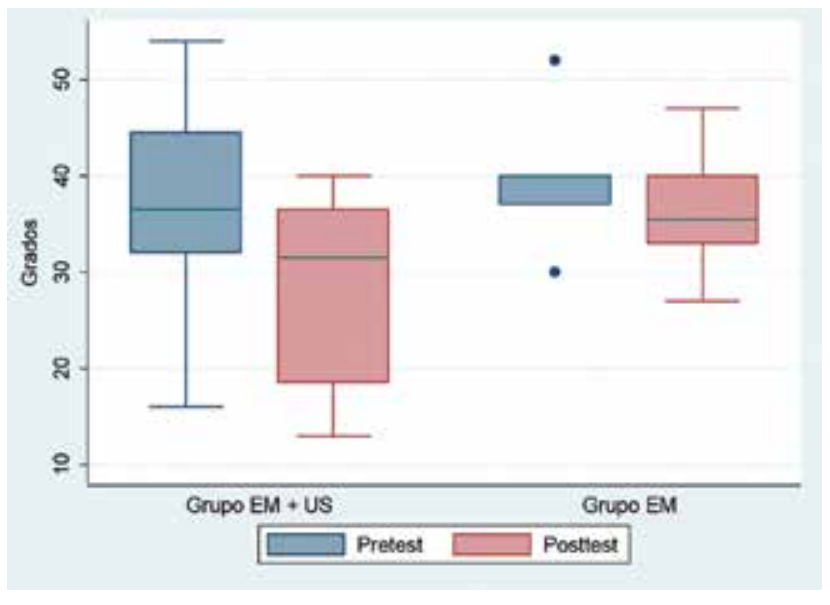

Figura 4. Arco de movilidad articular de extensión de rodilla con 90 grados de flexión de cadera antes y después de la intervención según grupo intervenido.

Se realizaron análisis de covarianza ANCOVA para determinar el efecto de las intervenciones en los grados de la evaluación posintervención ajustando por los grados de la evaluación inicial (Tabla 3).

Tabla 3. Medición final goniométrica de los isquiotibiales, ajustada por la medición inicial en el grupo EM + US y por actividad física

\begin{tabular}{lccc}
\hline & Delta & IC95\% & Valor $\mathbf{p}$ \\
\hline Grados postest & -5.8 & $-10,9 ;-0,67$ & 0,030 \\
$\begin{array}{l}\text { Grados postest ajustado } \\
\text { por actividad física }\end{array}$ & $-3,4$ & $-9,2 ; 2.4$ & 0,214 \\
\hline
\end{tabular}


Se puede observar un aumento estadísticamente significativo en los grados finales de las personas que hacían parte del grupo intervenido con EM + US, $-5,8$ (IC95\%: - 10,9;- 0,67 p=0,030) en comparación con el grupo de EM ajustando por puntaje inicial; sin embargo, cuando se ajustó además por los 4 niveles de actividad física, la diferencia entre grupos se reduce a $-3,4$ (IC95\% -9,2; 2,4 p=0,214) y pierde su significancia estadística, lo cual hace que esta característica se convierta en una variable confusora.

\section{DISCUSIÓN}

La combinación de modalidades de calentamiento y estiramiento terapéutico proveen un beneficio adicional para la ganancia de arco de movimiento en sujetos sanos. El estiramiento es ampliamente usado por atletas, entrenadores y terapeutas como un medio para mejorar, mantener o restaurar la flexibilidad muscular. Los objetivos del estiramiento pueden variar de acuerdo con la población, es útil para maximizar el desempeño en el deporte, prevenir lesiones, minimizar los cambios de flexibilidad relacionados con la edad, recuperar el rango de movimiento posterior a una lesión o proceso de inmovilización y disminuir el dolor muscular ${ }^{14}$.

Los mecanismos potenciales por los cuales los métodos de calentamiento pueden mejorar los resultados del estiramiento, se relacionan con el aumento de la temperatura tisular, aumento del flujo sanguíneo o disminución de la actividad muscular por medio de la relajación. Con respecto al aumento de la temperatura, podría influir directamente sobre el colágeno intramuscular, principalmente sobre el colágeno tipo I, que provee la resistencia pasiva al músculo, el cual se vuelve más extensible con el aumento de dicha temperatura. Adicionalmente, el aumento de temperatura puede disminuir la viscosidad del músculo, conllevando mayores ganancias en la longitud muscular con menores cargas $^{14}$.

A través de este trabajo se estudió el efecto inmediato de la combinación del ultrasonido como método de calentamiento y el estiramiento mantenido, por esta razón se desarrolló en una sola sesión, y las evaluaciones fueron realizadas antes e inmediatamente después de la intervención. Sin embargo, los datos no pueden ser confrontados con los presentados en la literatura, pues es común encontrar que los protocolos varían entre los estudios en relación con los parámetros de estiramiento y del ultrasonido.

En relación con los parámetros del estiramiento, tal como lo presenta el metaanálisis realizado por Nakano et al. ${ }^{14}$ en el que se revisaron 12 ensayos clínicos controlados, el número de sesiones varía entre una sesión hasta 18 sesiones, en un plazo máximo de 6 semanas. La duración del estiramiento es otra variable que cambia entre los estudios, pues el tiempo de aplicación varía de 15 segundos hasta 35 minutos, la intensidad del estiramiento no se reporta o se asocia al peso corporal. De igual forma, los parámetros del ultrasonido difieren o no son reportados, la frecuencia comúnmente empleada es de $1 \mathrm{MHz}$, no obstante, en otros estudios se emplea $3 \mathrm{MHz}$, la duración de la aplicación es entre 5 a 7 minutos.

El metaanálisis conducido por Nakano et al. ${ }^{14}$ demostró que tanto una sesión como múltiples sesiones de estiramiento mantenido y ultrasonido mejoran el rango de movimiento, comparado con el estiramiento mantenido aislado, lo cual se corresponde con lo encontrado en nuestro estudio, pues se puede concluir que una sola exposición al estiramiento combinado con el ultrasonido es suficiente para potenciar las ganancias en el rango de movimiento, así sean de pequeña magnitud.

En este estudio se escogió la aplicación de ultrasonido por ser una modalidad de calentamiento profundo, tal como lo demuestra la literatura, de acuerdo con la profundidad que logra el ultrasonido, es posible que el calentamiento alcance las fibras musculares, el tejido conectivo intramuscular (endomisio y perimisio), la vaina muscular (perimisio), el tejido adiposo y la piel, por lo cual se puede lograr mayor efecto sobre la extensibilidad del músculo. Sin embargo, en una revisión sistemática no se encontraron diferencias significativas entre los grupos de acuerdo con el método de aplicación de calor, lo cual puede ser explicado por diferencias entre los protocolos de estudio relacionados con modalidad de calentamiento, grupo muscular, género, edad y número de sujetos ${ }^{14}$.

Las revisiones sistemáticas han demostrado que las modalidades de calentamiento son útiles para potenciar la capacidad del estiramiento mediante sus efec- 
tos agudos y a largo plazo. Los mecanismos a través de los cuales los métodos de calentamiento pueden influenciar los efectos del estiramiento no son claros, pero podría ser explicado por: el aumento de la extensibilidad del tejido conectivo, la alteración de las propiedades visco elásticas del músculo, la adición de sarcómeras en serie y la alteración de la sensación de estiramiento, lo cual ha sido demostrado in vitro ${ }^{14}$.

En relación con los cambios plásticos de las fibras musculares se ha demostrado que en un programa de flexibilización el principal resultado es el aumento de las sarcómeras en serie, con el objetivo de aumentar la longitud muscular y por ende la extensibilidad de los tejidos. Existen diferencias entre la plasticidad de los tendones y los músculos, Stolov et al. ${ }^{8}$ compararon la curva de longitud tensión pasiva de la unión miotendinosa de los gastronemios de ratas y establecieron que el vientre muscular contribuye con el $95 \%$ de la longitud total de los gastronemios en contraste con el 5\% aportado por el tendón aisladamente, así, la longitud pasiva del músculo fue 19 veces mayor que la longitud del tendón bajo una carga progresiva, sin embargo, este estudio no pudo establecer la diferencia entre los cambios plásticos y elásticos, pues no se evaluó el aumento de la longitud posterior al retiro de la tensión.

Ahora bien, en la literatura autores como Lehmann et al. ${ }^{15}$ demostraron que el US puede generar un calentamiento profundo de los tejidos aumentando su extensibilidad, rango de movilidad y aumentando la longitud ganada con la misma fuerza de estiramiento, mientras que se disminuyen los riesgos de daño muscular, igualmente establecen que dicho aumento en la longitud muscular es mantenido más efectivamente si la fuerza de estiramiento es aplicada mientras la temperatura es elevada; sin embargo, en nuestro estudio el estiramiento no fue aplicado concomitantemente con el ultrasonido, sino se procedió inicialmente a calentar la zona y posteriormente a realizar el estiramiento mantenido mediante una técnica manual.

Wessling et al. ${ }^{2}$ realizaron un ensayo clínico controlado en 30 mujeres saludables con edades comprendidas entre 20 y 30 años, con el objetivo de demostrar que la combinación del ultrasonido y el estiramiento mantenido aumenta la extensibilidad del tríceps sural más que el estiramiento mantenido aplicado de forma aislada, en sus resultados cabe resaltar que el estiramien- to mantenido produjo un aumento de la dorsiflexión aproximadamente 1,3 grados mayor que la del grupo control ( $\mathrm{p}<0,001)$; de igual forma el estiramiento mantenido combinado con el ultrasonido produce un aumento en la flexión dorsal 20\% mayor que el tratamiento con estiramiento mantenido solo $(\mathrm{p}<0,001)$.

En contraste, Brodowicz et al. ${ }^{10}$ evaluaron 24 jugadores de género masculino, pertenecientes a un equipo de beisbol, comparando el efecto del estiramiento mantenido durante 20 minutos con aplicación de crioterapia, paquete caliente o estiramiento solo; los resultados evidencian que la aplicación de hielo provee mayor efecto a corto plazo en la flexibilidad de los isquiotibiales, que el paquete caliente o el estiramiento aislado; los resultados pueden ser atribuidos a una disminución de la retroalimentación propioceptiva posterior al estiramiento y aplicación de crioterapia que puede influir en un aumento del rango de movimiento. Aunque los resultados de este estudio son contradictorios con otros estudios que comprueban la mayor eficacia del paquete caliente; pueden relacionarse con la eficacia del frío para generar una disminución del reflejo de Hoffman o reflejo de estiramiento, que en teoría puede estar influenciado por el tiempo de estiramiento aplicado en el estudio.

Knight et al. ${ }^{16}$ realizaron una investigación para evaluar la efectividad de tres métodos de calentamiento sobre la extensibilidad de los plantiflexores. Los resultados demuestran que a la sexta semana los participantes del grupo que recibió ultrasonido continuo durante 7 minutos previo al estiramiento, lograron mayores aumentos en el rango de movilidad activo (6,20 grados) y pasivo (7,35 grados) de los dorsiflexores en comparación con los otros grupos.

Gersten citado por Wessling et al. ${ }^{2}$ demostró que la extensibilidad del tendón aumenta significativamente con la aplicación del ultrasonido entre $1 \mathrm{a} 3 \mathrm{~W} / \mathrm{cm}^{2}$, así mismo, la intensidad del US se encuentra en relación con la profundidad del tejido; si la profundidad es mayor de $8 \mathrm{~cm}$ se usa una intensidad de $1,5 \mathrm{~W} / \mathrm{cm}^{2}$. Lehman et al. ${ }^{15}$ demostraron que el calentamiento profundo producido por el ultrasonido de $1 \mathrm{MHz}$ de forma continua y con una intensidad de 1,0 a $2,5 \mathrm{~W} /$ $\mathrm{cm}^{2}$ es más efectivo para mejorar el rango de movilidad de la cadera que el calentamiento superficial en conjunto con ejercicio. Por esta razón, el ultrasonido 
con suficiente intensidad y duración para aumentar la temperatura puede aumentar la extensibilidad de los tejidos, disminuyendo el acortamiento muscular y aumentando el rango de movilidad cuando se aplica en combinación con técnicas de estiramiento con una duración de 5 a 10 minutos. Gersten demostró que las propiedades de plasticidad de los tendones aumentan a medida que aumenta la temperatura.

Las diferencias de nuestro estudio pueden estar relacionadas con las siguientes limitaciones: selección de una técnica de estiramiento manual, en la cual no es posible garantizar la tensión aplicada de forma homogénea para todos los participantes. Así mismo, el área de tratamiento con el ultrasonido podría no generar un calentamiento uniforme y efectivo de la zona de los isquiotibiales.

Los resultados obtenidos en este estudio son contradictorios a los encontrados en la literatura, posiblemente por falta de poder, debido a un tamaño de muestra muy pequeño, lo cual hace que los datos obtenidos no se puedan extrapolar a otras poblaciones y no sean generalizables. Por esta razón, este estudio se convierte en un reporte preliminar que sirva de base para futuros estudios que busquen demostrar la efectividad de diversas modalidades fisioterapéuticas encaminadas hacia la mejoría de la extensibilidad y flexibilidad muscular.

\section{CONCLUSIÓN}

Mediante la realización de este estudio se puede concluir que una sola exposición al estiramiento mantenido combinado con el ultrasonido es suficiente para potenciar las ganancias de pequeña magnitud en la extensibilidad de los músculos isquiotibiales en las mujeres adultas participantes, comparado con el estiramiento mantenido solo.

\section{REFERENCIAS}

1. Daza J. Evaluación clínico-funcional del movimiento corporal humano. Bogotá: Colomb Editor Médica Panam; 2007.

2. Wessling KC, Devane DA, Hylton CR. Effects of static stretch versus static stretch and ultrasound combined on triceps surae muscle extensibility in healthy women. Phys Ther. 1987;67(5):674-9.
3. Alter MJ. Ciência da flexibilidade. Brasil: Artmed; 1999.

4. Corbin CB, Dowell LJ. Concepts in physical education with laboratories and experiments. Dubuque: Wm.c. Brown company Published; 1977.

5. Wang K, McCarter R, Wright J, Beverly J, Ramirez-Mitchell R. Regulation of skeletal muscle stiffness and elasticity by titin isoforms: a test of the segmental extension model of resting tension. Proc Natl Acad Sci U S A. 1991;88(16):7101-5.

6. Kisner C, Colby LA. Ejercicio terapéutico: fundamentos y técnicas. Buenos Aires: Médica Panam; 2010.

7. Lieber RL. Estructura del músculo esquelético, función y plasticidad. Madrid: McGraw-Hill Interamericana; 2004.

8. Stolov WC, Weilepp Jr TG, Riddell WM. Passive length-tension relationship and hydroxyproline content of chronically denervated skeletal muscle. Arch Phys Med Rehabil. 1970;51(9):517-25.

9. Usuba M, Miyanaga Y, Miyakawa S, Maeshima T, Shirasaki Y. Effect of heat in increasing the range of knee motion after the development of a joint contracture: an experiment with an animal model. Arch Phys Med Rehabil. 2006;87(2):247-53. doi: 10.1016/j.apmr.2005.10.015.

10. Brodowicz GR, Welsh R, Wallis J. Comparison of stretching with ice, stretching with heat, or stretching alone on hamstring flexibility. J Athl Train. 1996;31(4):324-7.

11. Cameron MH. Physical agents in rehabilitation: from research to practice. Elsevier Health Sciences; 2012.

12. Norkin CC, White DJ. Measurement of joint motion: a guide to goniometry. Philadelphia: FA Davis; 2003.

13. Ainsworth BE, Bauman A, Benaziza H, Blair SN, Booth Ml, Craig CL et al. USA Spanish Version Translate 2003 3Long Last 7 Days Self-Administered (version of the IPAQ). International Physical Activity Questionnaire 2003.

14. Nakano J, Yamabayashi C, Scott A, Reid WD. The effect of heat applied with stretch to increase range of motion: a systematic review. Phys Ther Sport. 2012;13(3):180-8. doi: 10.1016/j.ptsp.2011.11.003.

15. Lehmann JF, Masock AJ, Warren CG, Koblanski JN. Effect of therapeutic temperatures on tendon extensibility. Arch Phys Med Rehabil. 1970;51(8):481-7.

16. Knight CA, Rutledge CR, Cox ME, Acosta M, Hall SJ. Effect of superficial heat, deep heat, and active exercise warm-up on the extensibility of the plantar flexors. Phys Ther. 2001;81(6):1206-14. doi: 10.1093/ptj/81.6.1206. 